\title{
A UTILIZAÇÃO REBEn COMO FONTE EM DOIS PROGRAMAS STRICTU SENSO NA CIDADE DO RIO DE JANEIRO, 1992 - 2001'
}

\author{
THE USE OF REBEn (BRAZILIAN JOURNAL OF NURSING) AS A SOURCE IN TWO STRICT \\ SENSU PROGRAM IN THE CITY OF RIO DE JANEIRO, 1992 -2001
}

\section{UTILIZACIÓN DE REBEn COMO FUENTE DE CONSULTA EN DOS PROGRAMAS STRICTU SENSO EN LA CIUDAD DE RIO DE JANEIRO, 1992 -2001}

Osnir Claudiano da Silva Junior ${ }^{2}$ Wellington Mendonça de Amorim ${ }^{3}$ Priscila Brigolini Porfírio 4

\begin{abstract}
RESUMO: Trata-se do estudo da utilização da REBEn em dissertações e teses de dois programas públicos no Rio de Janeiro. Os objetivos: investigar o impacto deste periódico na produção do conhecimento de enfermagem, no período de 1992 a 2001; e analisar comparativamente a utilização deste em dissertações e teses. Utilizou-se da análise de documentos, tendo como fonte as referências bibliográficas. Os dados foram analisados quantitativamente. O estudo demonstrou que a REBEn permanece como um veículo significativo para o conhecimento científico em enfermagem, aproximadamente $50 \%$ dos autores a citaram em dissertações e $60 \%$ a citaram em teses. A revisão de literatura foi numericamente menor em dissertações do que nas teses, e quanto ao uso em dissertações, destacou-se o ano de 1995.
\end{abstract}

PALAVRAS CHAVE: produção científica, enfermagem, REBEn

ABSTRACT: This paper is concerned with the use of REBEn (Brazilian Journal of Nursing) in dissertations and theses produced in two public programs in Rio de Janeiro. Objectives: 1) to investigate the impact of this journal in the production of the knowledge in the field of nursing from 1992 to 2001, and 2) to analyze, comparatively, the use of the journal in theses and dissertations. Document analysis was done, based on bibliographical references. Data were analyzed quantitatively. The study demonstrated that REBEn remains an important publication for the development scientific knowledge in the nursing field. Approximately $50 \%$ of the authors cite it in dissertations, and $60 \%$ cite it in theses. Literature revision was numerically smaller in dissertations compared to theses. Regarding theses, 1995 was the year in which there were more references from REBEn.

KEYWORDS: scientific production, nursing

RESUMEN: Se trata de un estudio sobre la utilización de la REBEn en disertaciones y tesis de dos programas públicos en Rio de Janeiro. Los objetivos: investigar el impacto de ese periódico en la producción del conocimiento de enfermería desde 1992 hasta 2001; y analizar comparativamente su utilización en disertaciones y tesis. Se utilizaron el análisis de documentos y una bibliografía adecuada. Los datos se analizaron cuantitativamente. El estudio demostró que la REBEn permanece como un vehículo significativo para generar conocimiento científico en enfermería, ya que cerca del $50 \%$ de los autores la mencionaron en disertaciones y el $60 \%$ en tesis. La revisión de literatura fue numéricamente menor en las disertaciones que en las tesis. La Revista alcanzó el mayor uso en consultas para disertaciones en 1995.

PALABRAS CLAVE: la producción científica, enfermería

Recebido em 31/08/2002

Aprovado em 27/09/2002

\footnotetext{
1 Este artígo é produto de uma oficina de iniciação científica em história da enfermagem, com alunas do curso de graduação, desenvolvido pelo Laboratório de Pesquisa em História de Enfermagem - LAPHE, da EEAP/UNIRIO. Colaboraram as alunas Alessandra Magalhães Vieira, Alexandra de Medeiros Aleixo, Anna Simone S. Reis, Juliana Fernandes da Costa, Rafaela de Oliveira Lopes da Silva, Renata da Silva Corrêa Canella, Ticiane de Oliveira, Úria Fernanda da Encarnação e Viviane Monteiro Faria.

2 Professor Doutor. em Enfermagem da EEAP/UNIRIO. Pesquisador do LAPHE.

${ }^{3}$ Professor Mestre em Enfermagem da EEAP/UNIRIO. Doutorando da EEAN/NUPHEBRAS. Pesquisador do LAPHE.

${ }^{4}$ Aluna do $4^{\circ}$ periodo do curso de graduação em enfermagem da EEAP/UNIRIO.
} 


\section{INTRODUÇÃO}

Ao que parece, a idéia de uma associação de enfermeiras e de uma revista chegou até nós através de Edith Magalhães Fraenkel, influenciada por Lilian Clayton, Diretora da Escola de Enfermagem do Hospital Geral da Filadélfia (Annaes de Enfermgem, 1934 p. 3-9), em curso de pós-graduação. Segundo Clayton para uma profissão se desenvolver seria necessário uma associação e uma revista. Logo após o Congresso Internacional no Canadá (1929), Fraenkel que lá representou a Associação, e em contatos com Rachel Haddock Lobo, Marina Bandeira de Oliveira e Célia Peixoto Alves, transmitiu-lhes a sugestão da criação de uma revista que foi acolhida com entusiasmo por estas tendo, a partir desse momento, o propósito da criação da revista quando regressaram ao Brasil (CARVALHO, 1976, $p$. 21, VIEIRA, 1995, p. 20, SANTOS et al., 1998, p. 306).

Ao longo de sete décadas a Revista Brasileira de Enfermagem (REBEn) tem contribuido fundamental e decisivamente na produção, divulgação, crítica e aplicação do conhecimento de enfermagem no Brasil. O presente estudo tem como objeto a utilização da REBEn como fonte na elaboração das dissertações de mestrado e teses de doutorados em dois programas públicos na cidade do Rio de Janeiro, tendo como marco inicial a defesa da $1^{\mathrm{a}}$ tese em um dos programas, em 1992.

Trata-se de um artigo elaborado em uma oficina de iniciação científica em História da Enfermagem, com alunas dos $4^{\circ}$ e $5^{\circ}$ períodos do curso de graduação em enfermagem da EEAP coordenada por dois pesquisadores do LAPHE durante $\circ 1^{\circ}$ semestre de 2002. Este trabalho se insere na linha de pesquisa em "A Profissionalização da Enfermagem no Brasil", do Laboratório de Pesquisa em História da Enfermagem (LAPHE), da Escola de Enfermagem Alfredo Pinto (EEAP), na Universidade do Rio de Janeiro (UNIRIO).

A importância deste estudo apoia-se no fato de ter a REBEn caráter nacional e internacional na divulgação da produção de pesquisas de enfermeiros (as), sendo o periódico mais antigo na área de enfermagem do país, classificado como Qualis Nacional A, de publicação regular no período estudado, com uma tiragem de 600 exemplares trimestrais, e ainda por pretender este artigo integrar a coletânea comemorativa do seu $70^{\circ}$ aniversário.

A fim de operacionalizar e de responder a questão que norteou o estudo "Em que proporção a REBEn foi utilizada como subsídio para elaboração de dissertações e teses em dois programas de pós-graduação strictu sensu no Rio de Janeiro?" foram traçados os seguintes objetivos: investigar preliminarmente o impacto desta publicação no conhecimento acadêmico entre 1992 a 2001; e analisar comparativamente a utilização desta em dissertações e teses nos dois programas.

Trata-se de uma pesquisa histórica com base na analise de documentos, que utilizou como fontes primárias referências bibliográficas de 150 dissertações de um programa e 103 teses de outro programa de pós-graduação strictu sensu, públicos federais, localizados na cidade do
Rio de Janeiro, no período compreendido entre a apresentação e defesa da primeira tese de doutorado em um dos programas (1992) até o ano anterior a presente pesquisa (2001). Utilizouse das cópias disponiveis e acessiveis nas duas bibliotecas públicas dos programas em questão. Para coleta dos dados foi elaborada uma ficha de registro das ocorrências de referências bibliográficas nas obras consultadas, onde destacamos as seguintes informações: Instituição do programa (escola/universidade) onde foi realizada a consulta; dissertação ou tese; nome do autor (a); título do trabalho; nome do orientador; ano de apresentação e defesa; e as referências da REBEn no trabalho, além de observações gerais. Na etapa atual da oficina de iniciação científica, utilizamos para tratamento dos dados a análise quantitativa, apresentada através de freqüência absoluta e percentual das referências, em tabelas e quadros. O presente artigo é o primeiro resultado desta investigação.

\section{DESENVOLVIMENTO}

$\mathrm{Na}$ organização dos dados, encontramos a distribuição e a frequência com que os artigos publicados pela REBEn foram utilizados na produção do conhecimento em enfermagem, através das dissertações e teses nos programas de mestrado e doutorado, no periodo estudado.

Tabela 1 - Número de referências da REBEn nas dissertações de mestrado em um programa público na cidade do Rio de Janeiro, no período de 1992 a 2001

\begin{tabular}{|c|c|c|c|c|c|}
\hline \multirow[t]{2}{*}{ Ano em curso } & \multirow{2}{*}{$\begin{array}{l}\text { Número de } \\
\text { dissertações }\end{array}$} & \multicolumn{2}{|c|}{ Utilizaram a REBEn } & \multicolumn{2}{|c|}{ Não utilizaram a REBEn } \\
\hline & & $\mathrm{N}^{0}$ & $\%$ & $N^{\circ}$ & $\%$ \\
\hline 1992 & 13 & 10 & 76,93 & 03 & 23,07 \\
\hline 1993 & 16 & 08 & 50,00 & 08 & 50,00 \\
\hline 1994 & 06 & 04 & 66,67 & 02 & 33,33 \\
\hline 1995 & 10 & 09 & 90,00 & 01 & 10,00 \\
\hline 1996 & 13 & 04 & 30,87 & 09 & 69,23 \\
\hline 1997 & 15 & 05 & 33,44 & 10 & 66,66 \\
\hline 1998 & 13 & 08 & 61,54 & 05 & 38,46 \\
\hline 1999 & 27 & 14 & 51,86 & 13 & 48,14 \\
\hline 2000 & 24 & 09 & 37,50 & 15 & 62,50 \\
\hline 2001 & 13 & 03 & 23,08 & 10 & 76,92 \\
\hline \multicolumn{6}{|l|}{ Total no } \\
\hline periodo & 150 & 74 & 49,34 & 76 & 50,66 \\
\hline
\end{tabular}

A análise da tabela 1 demonstrou que das 150 dissertações consultadas, ocorreu um equilibrio entre a utilização $(49,34 \%)$ e não utilização $(50,66)$ da REBEn; nos cinco primeiros anos do decênio variou de $90 \%$ a $30,87 \%$ o percentual de citações, já no lustro seguinte, houve uma variação de $61,54 \%$ a $23,08 \%$ com expressiva queda a partir de 1998 , atingindo $23,08 \%$ de citações, em 2001. Cabe destacar que o ano de maior utilização percentual (90\%) ocorreu em 1995 e o de menor utilização $(23,08 \%)$ foi o ano de 2001. 
Tabela 2 - Número de referências da REBEn nas teses de doutorado, em um programa público na cidade do Rio de Janeiro, no periodo de 1992 a 2001

\begin{tabular}{cccccc}
\hline Ano em curso & $\begin{array}{c}\text { Número de } \\
\text { teses }\end{array}$ & $\begin{array}{c}\text { Utilizaram a REBEn } \\
N^{\circ}\end{array}$ & $\begin{array}{c}\text { Não utilizaram a REBEn } \\
\%\end{array}$ & $N^{\circ}$ & $\%$ \\
\hline 1992 & 01 & 01 & 100,00 & 0 & 0,00 \\
1993 & 02 & 01 & 50,00 & 01 & 50,00 \\
1994 & 03 & 01 & 33,44 & 02 & 66,66 \\
1995 & 03 & 02 & 66,66 & 01 & 33,44 \\
1996 & 13 & 07 & 53,85 & 06 & 46,15 \\
1997 & 08 & 04 & 50,00 & 04 & 50,00 \\
1998 & 16 & 10 & 62,50 & 06 & 37,50 \\
1999 & 13 & 11 & 84,62 & 02 & 15,38 \\
2000 & 25 & 12 & 48,00 & 13 & 52,00 \\
2001 & 19 & 12 & 63,16 & 07 & 36,84 \\
Total no & & & & & \\
período & 103 & 61 & 59,22 & 42 & 40,78 \\
\hline
\end{tabular}

A análise da tabela 2 demonstrou que das 103 teses consultadas, $59,22 \%$ utilizaram a REBEn e $40,78 \%$ não utilizaram. $\mathrm{Na}$ análise do periodo, não é possivel identificar uma tendência nem de aumento, nem de diminuição, destacou-se apenas o ano de 1999 quando $84,62 \%$ da teses utilizaram referências dos artigos publicados na revista.

Quadro 1 - Distribuição do número de referências em cada dissertação e teses em dois programas na cida de do Rio de Janeiro, no período de 1992 a 2001

\begin{tabular}{|ccc|}
\hline Número de referências & Número de dissertações & Número de teses \\
\hline Nenhuma & 76 & 42 \\
1 a 3 & 47 & 39 \\
4 a 6 & 17 & 11 \\
7 a 9 & 06 & 04 \\
10 a 12 & 02 & 03 \\
13 a 15 & 02 & 03 \\
37 & 0 & 01 \\
Total (510 referências) & 150 & 103 \\
\hline
\end{tabular}

Neste quadro procurou-se demonstrar que os a produção acadêmica publicada na REBEn podem aparecer inúmeras vezes; houve 250 referências em 74 trabalhos de mestrados, com média de 3,38 por dissertação, e 260 referências em 61 trabalhos de doutorado, com uma média de 4,26 por tese.

\section{RESULTADOS}

Esse estudo possibilitou verificar a utilização da
Revista Brasileira de Enfermagem como referência para a construção de dissertações e teses em dois diferentes programas de pós-graduação strictu sensu, públicos na cidade do Rio de Janeiro.

A Revista é um veículo importante para o conhecimento científico, utilizado em cerca de $50 \%$ das dissertações de mestrado e $60 \%$ das teses de doutorado. As médias respectivas de citações são de 3,38 nas dissertações e 4,26 nas teses. Podemos considerar que estas diferenças de utilização se dão pelas características dos trabalhos desenvolvidos: a dissertação de mestrado requer uma revisão de literatura menor que a do que a exigida por uma tese de doutorado.

O ano de 1995 destaca-se como o ano do pico da utilização da REBEn nas dissertações de mestrado (90\%) e, em relação as teses de doutorado, o ano de maior incidência foi 1999 (84,62\%).

Cabe destacar que a biblioteca do curso de mestrado não manteve assinatura da REBEn e os poucos exemplares existentes nesta biblioteca foram doados, o que representa um grau de defasagem temporal que dificulta ou compromete a atualidade do produto acadêmico.

\section{CONSIDERAÇÕES FINAIS}

A revista continua sendo importante instrumento para a produção de dissertações e teses. É sensivelmente mais utilizada nas teses que nas dissertações. O programa de doutorado tem assinatura regular e o programa de mestrado não possui assinatura regular da revista, o que pode ter influenciado na utilização da mesma.

Para o grupo de alunas de enfermagem, foi importante elaborar este trabalho devido a aproximação com a história da REBEn, a possibilidade de manipular 
dissertações e teses, e a participação na produção de um trabalho científico em História da Enfermagem.

O trabalho de pesquisa continua, sendo levantadas outras questões como por exemplo: a manifesta tendência de queda na utilização da REBEn nas dissertações de mestrado, principalmente, no que se refere as temáticas publicadas e sua relação com os trabalhos produzidos

\section{SUGESTÕES}

Às Bibliotecas que mantenham assinatura regular da REBEn dado seu caráter pioneiro e atual na produção científica da profissão e na qualidade já obtida pelo periódico, um patrimônio da Enfermagem Brasileira.

\section{REFERÊNCIAS BIBLIOGRÁFICAS}

ANNAES DE ENFERMAGEM. Em memória. S Lilian Clayton. Annaes de Enfermagem, Ano II, n. 2, p. 3-9, abr. 1934.

CARVALHO, A. de C. Associação Brasileira de Enfermagem (1926-1976): Documentário. Rio de Janeiro: ABEn, 1976. 514p.

ECO, U. Como se faz uma tese. 17. ed. São Paulo: Perspectiva, 2002.

SANTOS, I. dos. et al. Revista Brasileira de Enfermagem - uma história de resistência. Rev. Bras. de Enfermagem, Brasilia, v. 51, n.2, p.305-320, abr./jun. 1998.

VIEIRA, T. T. Catálogo Analítico: Nexos EEAN/ABEn (1925-1975), 1995. 1 CD-ROM. 